\title{
Serious Games and Participatory Research in Public Health
}

\author{
Ann Borda \\ Health and Biomedical Informatics \\ Centre \\ Melbourne Medical School \\ University of Melbourne \\ Australia \\ aborda@unimelb.edu.au
}

\author{
Andreea Molnar \\ Department of Computer Science \\ and Software Engineering \\ Swinburne University of \\ Technology \\ Australia \\ amolnar@ @ swin.edu.au
}

\author{
Patty Kostkova \\ UCL IRDR Centre for Digital \\ Public Health in Emergencies \\ (dPHE) \\ University College London \\ United Kingdom \\ p.kostkova@ucl.ac.uk
}

\begin{abstract}
This survey study considers the state of the art of participatory research approaches using serious games to improve public health. It provides perspectives on existing research and future directions.
\end{abstract}

\section{KEYWORDS}

Participatory research; Public Health; Serious Games

\section{ACM Reference format:}

Ann Borda, Andreea Molnar and Patty Kostkova. 2019. Serious Games and Participatory Research in Public Health. In DPH'19: 9th International Digital Public Health Conference, November 20-23, 2019, Marseilles, France. 1page. https://doi.org/10.1145/3357729.3357762

\section{Introduction}

Among the grand challenges of digital health, the emergence of serious games was identified for its potential as a technologyenabled intervention in the improvement of health and wellbeing of individuals and wider populations [4]. Serious games can be defined as digital game technologies that serve a 'serious' purpose, for instance, imparting knowledge or skills. There is an exponential rise in the last decade of such games in the healthcare context, especially in the rapid take-up of mobile and smartphone (mHealth) opportunities and increasing technological literacy [1]. Serious games have targeted different segments of the population, such as games aimed to teach children about microbes and responsible hygiene, e.g. MicrobeQuest! [4], games targeted to the education of healthcare professionals to reduce errors and support clinical decision-making without risk to patients [5], and promoting healthy lifestyles through the use of gamification and positive reinforcement techniques, for example, in the context of improving self-management of chronic conditions [3].

\section{Participatory Health and Serious Games}

To date, there is a gap in our understanding of participatory forms of digital public health and the role of serious games. In the results of a thematic literature review, there is a relevant trend in the mainstreaming of serious games as a design approach in public health citizen science [7]. Citizen science generally relates to active participation in research projects by members of the general public with no formal training in the field of research concerned.
Participation can include data collection, processing and analysis tasks. Gamified tasks have shown to improve accuracy of disease identification, or motivate gamers to perform complex pattern recognition as in the protein folding game Foldit, or support global disease surveillance as in the game SpotLab which uses mobile phones turned into low-cost microscopes to provide collective diagnosis of malaria [3, 7, 8]. In a public health landscape, gamified citizen science can also strengthen localised participatory epidemiology, such as neighbourhood mapping of health-related behaviours using mobile apps on which community interventions can then be based [2].

An acknowledged limitation, and opportunity, in the current study is identifying and evaluating different types of public participation, e.g. participant involvement in serious game development, and/or in its education and public health intervention goals.

\section{Conclusion}

Within the scope of this study, serious game use and design could potentially support digitally enabled public health outcomes through public participation opportunities, such as citizen science and edutainment. Future work needs to address the extent and realworld deployment of serious games aligned with appropriate forms of participation in a digital public health educational context.

\section{REFERENCES}

[1] S Arnab, I Dunwell, and K Debattista. eds. 2012. Serious Games for Healthcare: Applications and Implications. Hershey, PA: IGI Global.

[2] M Bach, S Jordan, S Hartung, C Santos-Hovener, and M.T Wright. 2017. Participatory epidemiology: the contribution of participatory research to epidemiology. Emerg Themes Epidemiol 14, 2.

[3] N Charlier, N Zupancic, S Fieuws, K Denhaerynck, B Zaman, and P Moons. 2016. Serious games for improving knowledge and self-management in young people with chronic conditions: a systematic review and meta-analysis. J Am Med Inform Assoc, 23, 230-239.

[4] P Kostkova. 2018. Disease surveillance data sharing for public health: the next ethical frontiers. Life Sciences, Society and Policy,14, 1, Article 16.

[5] R Wang, S DeMaria Jr, A Goldberg and D Katz. 2016. A Systematic Review of Serious Games in Training Health Care Professionals. Simul Healthcare 11, 1 (2016), 41-51.

[6] A Molnar and P Kostkova. 2018. Learning about Hygiene and Antibiotic Resistance through Mobile Games: Evaluation of Learning Effectiveness. In DH' 18: 2018 International Digital Health Conference, April 23-26, Lyon, France, 95 99.

[7] M Ponti, T Hillman, C Kullenberg, and D Kasperowski. 2018. Getting it Right or Being Top Rank: Games in Citizen Science. Citizen Science: Theory and Practice, 3, 1 (2018), 1-12.

[8] K Wazny. 2018. Applications of crowdsourcing in health: an overview. Journal of Global Health, 8, 1 (2018), 010502. 